Luz Dahiana Alfaro Carballido' Carmen Rosa García Rupaya ${ }^{2}$

${ }^{1}$ Cirujano - Dentista

Cedicina

y Cirugía Buco Maxilofacial. Facultad de

Estomatología. Universidad Peruana Cayetano Heredia.

\section{Correspondencia}

Carmen Rosa García Rupaya Honorio Delgado 430, Lima 31 - Perú Teléfono: $5781497 / 999644302$ e-mail: camen.garcia@upch.pe

Recibido : 25 de enero de 2011

Aceptado : 15 de marzo de 2011

\title{
Percepción del proceso de consen- timiento informado en pacientes de la Clínica Estomatológica Central de la Universidad Peruana Cayetano Heredia
}

Alfaro-Carballido LD, García-Rupaya CR. Percepción del proceso de consentimiento informado en pacientes de la Clínica Estomatológica Central de la Universidad Peruana Cayetano Heredia. Rev Estomatol Herediana. 2011; 21(1):5-12.

\section{RESUMEN}

El propósito del estudio fue evaluar el proceso de consentimiento informado en pacientes de la Clínica Estomatológica Central de la UPCH, para lo cual se elaboró y validó un cuestionario que permitió evaluar el (PCI) en cuatro dimensiones: información, entendimiento, autonomía y valores. La aplicación final del instrumento fue en una muestra de 90 pacientes, la cual se cálculo del estudio piloto. De acuerdo a los datos obtenidos por el cuestionario, la mayoría de pacientes respondió que "Si" firmó un documento de consentimiento informado (85\%); al evaluar el (PCI), el entendimiento de la información y los valores del profesional fue calificado como muy favorable, mientras que la información y autonomía solo como favorable. Los resultados indicaron que a mayor número de citas, la información se percibe de forma más favorable $(\mathrm{p}=0,043)$ y que a mayor grado de instrucción la comprensión y entendimiento es mejor $(\mathrm{p}=0,000)$; de la misma forma, que si el profesional no tiene parentesco con el paciente $(p=0,000)$. La autonomía de los pacientes fue indistinta del género, edad y grado de instrucción; mas si se encontró diferencia entre pacientes y tutores $(p=0,020)$; el lugar de residencia también fue significativo para esta dimensión $(\mathrm{p}=0,023)$. En conclusión, el proceso de consentimiento informado se viene dando de forma favorable, lo que quiere decir que los pacientes tienen una percepción clara y precisa, sin embargo, se deben mejorar aspectos relacionados con la información y respeto de la autonomía del paciente.

Palabras clave: CONSENTIMIENTO INFORMADO / AUTONOMÍA PERSONAL / CUESTIONARIOS / ODONTOLOGÍA.

Perception of the informed consent process in patients from the Central Dental Clinic at Universidad Peruana Cayetano Heredia

ABSTRACT

The purpose of this study was to evaluate the informed consent process in patients of the Central Dental Clinic UPCH, for which was developed and validated a questionnaire to evaluate the (ICP) in four dimensions: information, understanding, autonomy and values. The final application of the instrument was in a sample of 90 patients, which was calculated from the pilot study. According to data obtained by the questionnaire, the majority of patients responded that they signed a document of informed consent (85\%); when the ICP was evaluated, the understanding of the information and professional values was rated as very favorable, while the information and autonomy were rated as just favorable. The results indicated that the higher number of appointments, the information is perceived more favorable $(\mathrm{p}=0.043)$ and that the higher education level, the comprehension and understanding is better $(\mathrm{p}=0.000)$ in the same way, if the professional is not related to the patient $(p=0.000)$. The autonomy of the patients was indiscriminate regarding the gender, age and educational level, but a difference was found between patients and tutors $(\mathrm{p}=0.020)$, the place of residence was also significant for this dimension $(\mathrm{p}=0.023)$. In conclusion, the informed consent process has been going in a favorable way, which means that patients have a clear and accurate perception, however, should improve aspects relative to information and respect for patient autonomy.

Key words: INFORMED CONSENT / PERSONAL AUTONOMY / QUESTIONNAIRES DENTISTRY.

\section{Introducción}

Una de las principales preocupaciones de la bioética moderna, trata de la relación médico-paciente, la cual es pieza clave y elemental del proceso de consentimiento informado (1-5). La relación odontólogopaciente ha pasado de ser un sistema vertical, en el que el cirujano dentista era el centro del sistema, que de forma paternalista tomaba las decisiones, en muchos casos de forma unilateral, e indiscutible debido al alto conocimiento especializado relacionado con el tratamiento de enfermedades dentales siendo el paciente receptor pasivo de los cuidados; a otro horizontal, donde el paciente es el axis y centro del sistema sanitario, el cual debe ser respetado como agente autónomo y como tal debe de participar activa- mente en conjunto con el profesional y tomar sus propias decisiones (4).

Como respuesta de este cambio de mentalidad surge el concepto de consentimiento informado, definido como el proceso por el cual a un paciente se le provee la suficiente información para tomar una decisión informada y razonada del procedimiento clínico. Este proceso se da 
sin ninguna coacción o fraude, e incluye explicar la necesidad del tratamiento, sus beneficios, alternativas, costos, riesgos y complicaciones o la no aceptación del tratamiento de la condición diagnosticada; este proceso debe darse con claridad, en un lenguaje comprensible y de forma oportuna (5).

Con la introducción del documento de consentimiento informado, se ha inducido al profesional de la salud a pensar, que sus obligaciones respecto a los pacientes y a su organización se cumplían, si conseguían "la firma" del paciente, sin embargo, el formulario firmado no garantiza que el derecho a la información haya sido realmente respetado. Por lo tanto, este proceso no debe verse como una actividad irrelevante, en la que el profesional consigue la autorización del paciente solo para protegerse de posibles procesos legales que por ventura podrían ocurrir (6).

Conocer la percepción de los propios pacientes sobre el proceso de consentimiento informado resulta importante porque permite evaluar el desempeño de la institución en este aspecto y elaborar pautas que orienten hacia una mejora en la calidad de la atención y satisfacción de los pacientes.

El propósito de esta investigación fue evaluar la opinión de los pacientes sobre el proceso de consentimiento informado en la Clínica Estomatológica Central de la UPCH, en relación a cuatro dimensiones: información, entendimiento, autonomía y valores; por otro lado también se planteó evaluar si determinadas variables como: persona responsable de la información, sexo, edad, grado de instrucción, lugar de residencia, especialidad en que se atiende, tratamiento, número de citas, tiempo de atención en la clínica y parentesco con el profesional, pueden variar o condicionar este proceso, estableciendo diferencias en la práctica real del consentimiento informado.

\section{Material y métodos}

El tamaño de la muestra, de tipo probabilística, fue de 90 pacientes y fue obtenida a partir de los resultados del estudio piloto. Participaron del estudio los pacientes y tutores de los menores de edad, que acudieron a la clínica durante el periodo de junio-agosto de 2009 y se excluyeron aquellos pacientes que llegaban por primera vez a la clínica y los que refirieron su negativa a responder al cuestionario.

El método empleado fue la encuesta estructurada; la técnica consistió en aplicar un cuestionario autoadministrado, donde se registró: lugar y fecha de obtención de la información, datos generales del paciente, características de la atención odontológica y 33 ítems de respuesta múltiple de acuerdo a la escala de Likert.

El proceso de construcción y validación del instrumento se realizo mediante un juicio de expertos que verifico la validez del contenido y constructo aplicando la $\mathrm{V}$ de Aiken $(0,99)$, luego se evaluó la confiabilidad obteniendo resultados del Alfa de Crombach $(0,73)$ y el análisis Factorial, esta secuencia ha sido descrita por diversos autores para la validación de cuestionarios $(7,8)$.

Se obtuvo la autorización del comité de ética de la universidad y el permiso de las autoridades de la unidad de estudio previo a la ejecución de la investigación.

El cuestionario fue aplicado al azar basado en el cumplimiento de los criterios de inclusión, exclusión y la aceptación del usuario de parti- cipar en el estudio previo consentimiento informado.

Los cuestionarios fueron entregados a los pacientes en las salas de espera de la Clínica, se estableció un cronograma y horarios para su aplicación y una vez terminado se verificó que todas las preguntas estuvieran resueltas para evitar perdida de datos.

Para el almacenamiento y procesamiento de la información, se creó una base de datos utilizando el programa Excel, se codificaron los datos y se asignó puntajes a la escala de Likert.

El análisis estadístico se realizó utilizando el programa estadístico computarizado SPSS 15 y se inició describiendo la información recolectada mediante tablas de distribución de frecuencias y gráficos de barras. Para evaluar el proceso de consentimiento informado en sus cuatro dimensiones primero se asignó valores numéricos a la escala de Likert siendo: totalmente de acuerdo (5), de acuerdo (4), ni en acuerdo ni en desacuerdo (3), en desacuerdo (2). Totalmente en desacuerdo (1). Se sacó un promedio final por cada dimensión de análisis y posteriormente se recodificó la información como muy favorable (5), favorable (4) y desfavorable $(3,2,1)$. Para su posterior presentación en tablas de contingencia.

Finalmente para determinar la relación del proceso de consentimiento informado de acuerdo a sus dimensiones: Información, Entendimiento, Autonomía y Valores y cada covariable, se utilizó la prueba de chi cuadrado.

\section{Resultados}

De las 90 encuestas respondidas, 63(70\%) correspondieron a mujeres y $27(30 \%)$ a varones, la edad promedio de los encuestados fue de 
40.55 años de edad. En relación a la persona que respondió el cuestionario, 49 (54\%) eran pacientes y 41(46\%) apoderados. Con respecto al grado de instrucción, la mayor parte de la muestra, tenía estudios universitarios (58\%), seguido de educación técnica (16\%) y secundaria (23\%), mientras que sólo un pequeño porcentaje (3\%) tenía solo educación primaria. El servicio que tuvo mayor número de pacientes entrevistados fue el de ortodoncia con un $29 \%$. Se observó que la mayoría de pacientes (58\%) tenía más de 6 citas (Tabla 1).

Se realizó el análisis descriptivo de las respuestas dadas considerando como alternativas de respuesta: totalmente de acuerdo, de acuerdo, ni en acuerdo ni en desacuerdo, en desacuerdo.

En relación al ítem final acerca de la aplicación del consentimiento informado, el $85.6 \%$ de los pacientes recuerdan haber firmado el documento de consentimiento informado (Fig. 1).

Al evaluar el proceso de consentimiento informado en sus cuatro dimensiones se encontró que hay una percepción muy favorable de los

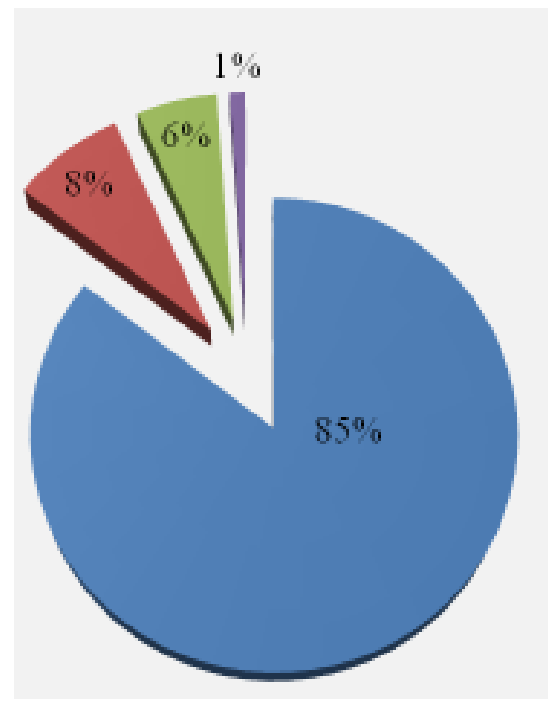

valores del profesional y entendimiento de la atención recibida en la clínica; a diferencia de la información recibida y autonomía del paciente en la toma de decisiones para procedimientos clínicos, que fue percibida solo como favorable (Fig. 2).

Al analizar la dimensión información según covariables, se encontró relación con el número de citas (Tabla 2).

Tabla 1. Descripción de la muestra de estudio de pacientes que acuden a la CEC-UPCH.

\begin{tabular}{lcc}
\hline & N & $\%$ \\
\hline Persona que responde & 49 & 54 \\
Paciente & 41 & 46 \\
Apoderado & 27 & \\
Sexo & 63 & 30 \\
Masculino & 40,55 & \\
Femenino & & \\
Edad promedio en años & 52 & 58 \\
Grado de instrucción & 14 & 16 \\
Universidad & 21 & 23 \\
Técnica & 3 & 3 \\
Secundaria & & \\
Primaria & 26 & 29 \\
Servicio & 17 & 19 \\
Ortodoncia & 17 & 19 \\
Endodoncia & 17 & 19 \\
Odontopediatría & 9 & 10 \\
Rehabilitación & 4 & 21 \\
Cirugía & & \\
Periodoncia & & 58 \\
Número de citas & 52 & \\
6 a más citas & 19 & \\
3 a 5 citas & 90 & \\
1 a 2 citas & & \\
Total & & \\
\hline
\end{tabular}

Fig. 1. Aplicación del consentimiento informado en los pacientes que acuden a la CEC-UPCH.
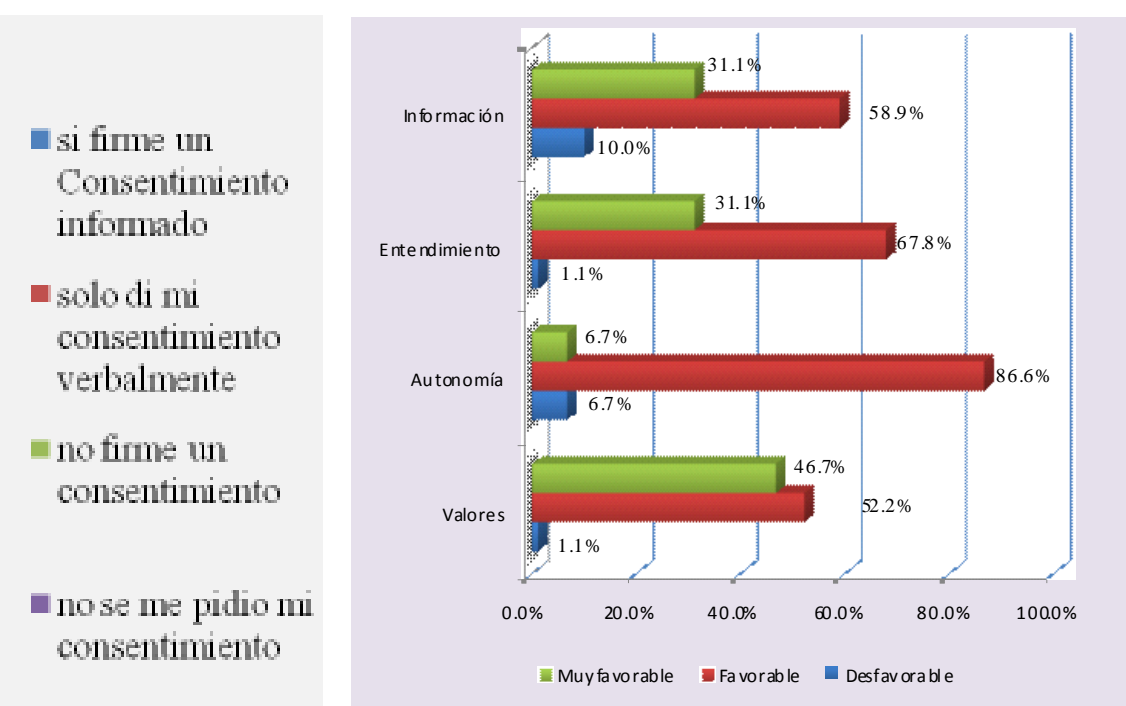

Fig. 2. Evaluación del proceso de consentimiento informado según dimensiones: información, entendimiento, autonomía y valores en pacientes que se atienden en la CEC-UPCH. 
Al relacionar las covariables con la dimensión entendimiento y la información recibida, se encontró diferencias significativas con el grado de instrucción del paciente y parentesco con el profesional (Tabla 3).

Con respecto a la autonomía del paciente en la toma de decisiones para procedimientos clínicos y las covariables, se encontró diferencia entre pacientes y tutores, teniendo los pacientes una actitud más favorable con respecto a su autonomía (Tabla 4).

Al evaluar la dimensión de los valores del profesional no se encontraron diferencias significativas para ninguna covariable, sin embargo se aprecia que el rango de respuestas esta entre muy favorable y favorable (Tabla 5).

\section{Discusión}

Se encontró que la mayoría de pacientes refieren haber firmado un documento de consentimiento informado $(85.6 \%)$ y un $7.8 \%$ haber dado su consentimiento de forma verbal, siendo estos resultados bastante favorables a comparación de los encontrados por el estudio de Guix et al. (9).

\section{Dimensión Información:}

En general los participantes, percibieron la información recibida en la Clínica como favorable.

Los datos obtenidos en la encuesta permiten observar que la mayoría, está de acuerdo en que se les informó de sus derechos y obligaciones como paciente y que se les brindó la información de forma clara, suficiente y oportuna, como también de los beneficios del tratamiento y sus distintas opciones; no obstante, casi la mitad de los participantes preferirían que se utilicen: cartillas de motivación, trípticos, imágenes, gráficos, etc., para una mejor información.

En porcentajes similares con lo encontrado por Guix et al. (9), los pacientes preferirían que se haga mayor énfasis al explicar los riesgos y posibles complicaciones del procedimiento, como también su derecho a negarse al mismo.

Por otra parte no se encontró diferencias en la percepción de la información según los factores característicos de los participantes, por el contrario, Benedetti et al. (10) encontraron diferencias en relación al grado de instrucción, lo que indica según el autor que a mayor grado de educación recuerdan mejor la in-

Tabla 2. Resultado de la Dimensión Información según covariables.

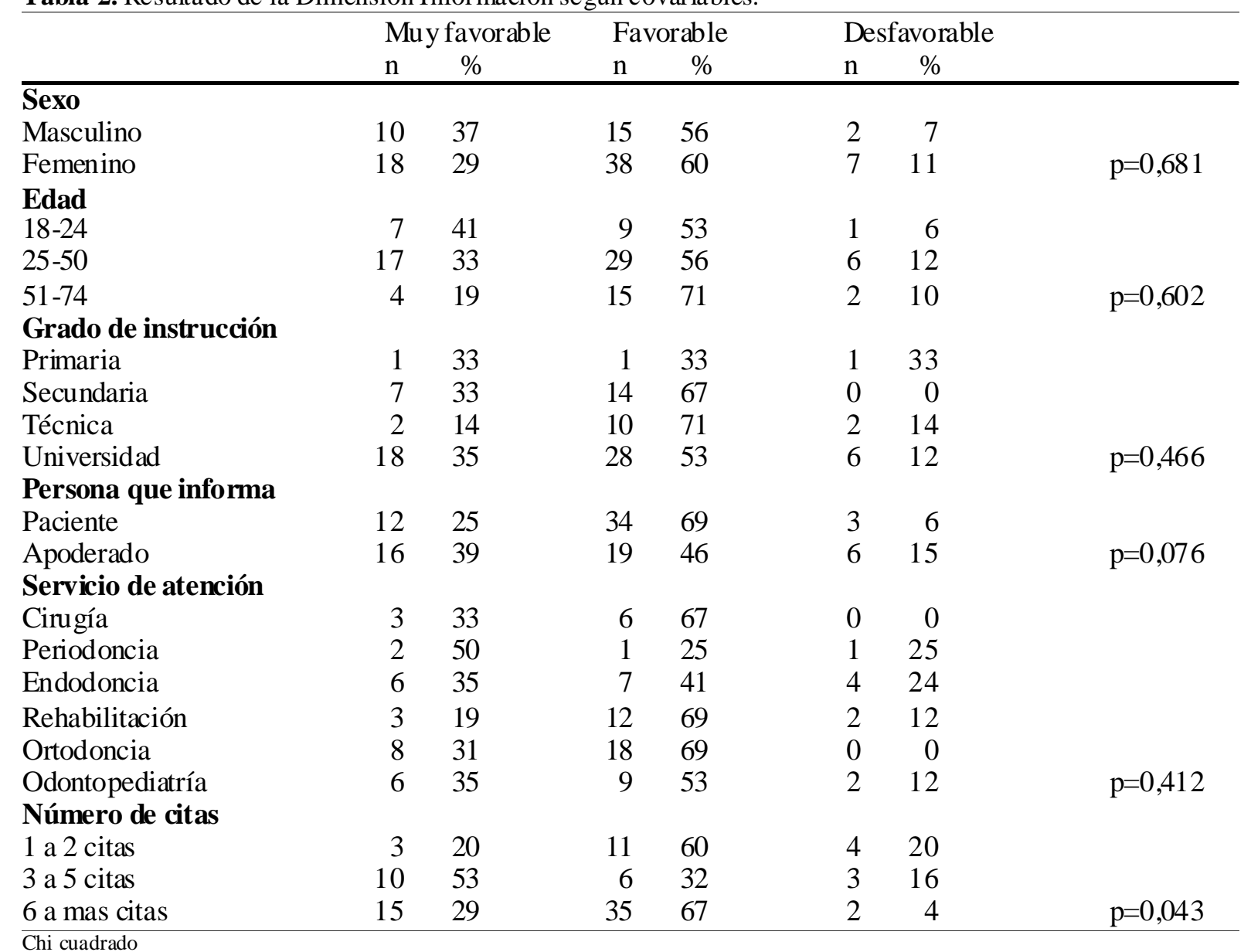


formación brindada; esta diferencia podría ser debido a que en nuestro estudio la mayor proporción de entrevistados tiene educación superior. La percepción de la información no tuvo relación con el servicio en el cual se atendió el paciente, sin embargo se encontró asociación en el número de citas, ya que a mayor número de citas asistidas, hubo una mejor percepción de la información por parte de los pacientes.

Dimensión Entendimiento y comprensión de la información recibida:
Para que el paciente pueda tomar una decisión pensada e informada, la comprensión de debe ser óptima. Los resultados de la encuesta mostraron que el grado de entendimiento y comprensión de la información fue muy favorable.

En general los pacientes refirieron no tener dificultad en comprender los documentos impresos proporcionados, ni con el lenguaje con que les habla el profesional; pero si les gustaría que se les motive a hacer preguntas para ampliar sus conocimientos sobre el tratamiento; simi- lar a los hallazgos de Simón et al. (6) donde una gran parte de pacientes hospitalizados en el hospital universitario Virgen de las Mercedes en Granada afirman con frecuencia quedarse con dudas y desearían preguntar más para ampliar la información.

Al igual que el estudio de Guix et al. (9) y Mortensen et al. (11) se encontró que hubo menor comprensión por parte de los pacientes con menor grado de instrucción, por lo cual el profesional debe incidir más en la resolución de dudas e incentivo de

Tabla 3. Resultado de la Dimensión Entendimiento según covariables.

\begin{tabular}{|c|c|c|c|c|c|c|c|}
\hline & \multicolumn{2}{|c|}{ Muy Favorable } & \multicolumn{2}{|c|}{ Favorable } & \multicolumn{2}{|c|}{ Desfavorable } & \\
\hline & $\mathrm{n}$ & $\%$ & $\mathrm{n}$ & $\%$ & $\mathrm{n}$ & $\%$ & \\
\hline \multicolumn{8}{|l|}{ Sexo } \\
\hline Masculino & 7 & 36 & 20 & 74 & 0 & 0 & \\
\hline Femenino & 21 & 33 & 41 & 65 & 1 & 2 & $\mathrm{P}=0,610$ \\
\hline \multicolumn{8}{|l|}{ Edad } \\
\hline $18-24$ & 7 & 41 & 10 & 59 & 0 & 0 & \\
\hline $25-50$ & 15 & 29 & 37 & 71 & 0 & 0 & \\
\hline $51-74$ & 6 & 29 & 14 & 67 & 1 & 5 & $\mathrm{P}=0,372$ \\
\hline \multicolumn{8}{|l|}{ Grado de instrucción } \\
\hline Primaria & 0 & 0 & 2 & 67 & 1 & 33 & \\
\hline Secundaria & 9 & 43 & 12 & 57 & 0 & 0 & \\
\hline Técnica & 5 & 36 & 9 & 64 & 0 & 0 & \\
\hline Universidad & 14 & 28 & 38 & 73 & 0 & 0 & $\mathrm{P}=0,000$ \\
\hline \multicolumn{8}{|l|}{ Persona que informa } \\
\hline Paciente & 17 & 35 & 32 & 65 & 0 & 0 & \\
\hline Apoderado & 11 & 27 & 29 & 71 & 1 & 2 & $\mathrm{P}=0,420$ \\
\hline \multicolumn{8}{|l|}{ Servicio de atención } \\
\hline Cirugía & 2 & 22 & 6 & 67 & 1 & 11 & \\
\hline Periodoncia & 1 & 25 & 3 & 75 & 0 & 0 & \\
\hline Endodoncia & 5 & 29 & 12 & 71 & 0 & 0 & \\
\hline Rehabilitación & 9 & 50 & 8 & 50 & 0 & 0 & \\
\hline Ortodoncia & 5 & 19 & 21 & 21 & 0 & 0 & \\
\hline Odontopediatría & 6 & 35 & 11 & 65 & 0 & 0 & $\mathrm{P}=0,188$ \\
\hline \multicolumn{8}{|l|}{ Numero de citas } \\
\hline 1 a 2 citas & 6 & 40 & 13 & 60 & 0 & 0 & \\
\hline 3 a 5 citas & 7 & 37 & 11 & 58 & 1 & 5 & \\
\hline 6 a mas citas & 15 & 29 & 37 & 71 & 0 & 0 & $\mathrm{P}=0,344$ \\
\hline \multicolumn{8}{|l|}{ Parentesco con el operador } \\
\hline Ninguna & 28 & 32 & 59 & 68 & 0 & 0 & \\
\hline Familiar & 0 & 0 & 1 & 100 & 0 & 0 & \\
\hline Conocido & 0 & 0 & 1 & 50 & 1 & 50 & $\mathrm{P}=0,00$ \\
\hline
\end{tabular}

Chi cuadrado 
preguntas en este tipo de pacientes y brindar la explicación en términos sencillos para conseguir una mejor comprensión del tema.

Se encontró también diferencia en el entendimiento de los pacientes que no tenían relación de parentesco con el profesional, siendo mejor, que la de los mismos familiares y conocidos, lo cual obedecería a que por el grado de amistad y confianza la explicación sobre los procedimientos sería menor.

Dimensión Autonomía:

La autonomía de los pacientes en la toma de decisiones para realizar procedimientos clínicos fue favorable.

La mayoría de pacientes firmó un documento de autorización en general (91\%) y un documento de autorización antes de recibir el tratamiento de la especialidad (84\%), siendo éstos mejores resultados que los encontrados por Guix et al (9) en 1999, donde solo el $61 \%$ de los pacientes referían haber firmado el documento de consentimiento informado; lo cual refleja que en esta época el uso del consentimiento informado ha cobrado mayor importancia y su aplicación es más frecuente, tanto en la práctica médica como odontológica y sus especiali- dades.

Asimismo los pacientes perciben que no se sienten presionados por el profesional en la toma de decisiones y que se les brinda las alternativas de tratamiento según sus costos.

Al igual que lo observado por Guix et al. (9) en el área médica hospitalaria, en un porcentaje menor los pacientes prefieren implicar a un familiar en la toma de decisiones.

Así, también del total de pacientes, el 31\% consulta en internet antes de elegir una alternativa de tratamiento, mientras que el $63 \%$ consulta por otras fuentes, lo cual concuerda con el estudio de Silva (12)

Tabla 4. Resultado de la Dimensión Autonomía según covariables.

\section{Muy Favorable Favorable Desfavorable}

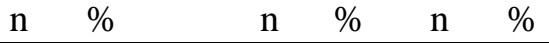

\begin{tabular}{|c|c|c|c|c|c|c|c|}
\hline \multicolumn{8}{|l|}{$\overline{\text { Sexo }}$} \\
\hline Masculino & 3 & 11 & 24 & 89 & 0 & 0 & \\
\hline Femenino & 3 & 5 & 54 & 86 & 6 & 10 & $\mathrm{p}=0,154$ \\
\hline \multicolumn{8}{|l|}{ Edad } \\
\hline $18-24$ & 2 & 12 & 15 & 88 & 0 & 0 & \\
\hline $25-50$ & 2 & 4 & 45 & 87 & 5 & 10 & \\
\hline 51-74 & 2 & 10 & 18 & 86 & 1 & 5 & $\mathrm{p}=0,482$ \\
\hline \multicolumn{8}{|c|}{ Grado de instrucción } \\
\hline Primaria & 0 & 0 & 3 & 100 & 0 & 0 & \\
\hline Secundaria & 3 & 14 & 16 & 76 & 2 & 10 & \\
\hline Técnica & 1 & 7 & 13 & 93 & 0 & 0 & \\
\hline Universidad & 2 & 4 & 46 & 88 & 4 & 8 & $\mathrm{p}=0,793$ \\
\hline \multicolumn{8}{|c|}{ Persona que informa } \\
\hline Paciente & 3 & 6 & 46 & 94 & 0 & 0 & \\
\hline Apoderado & 3 & 7 & 32 & 78 & 6 & 15 & $\mathrm{p}=0,020$ \\
\hline \multicolumn{8}{|c|}{ Servicio de atención } \\
\hline Cinugía & 0 & 0 & 9 & 100 & 0 & 0 & \\
\hline Periodoncia & 0 & 0 & 3 & 75 & 1 & 25 & \\
\hline Endodoncia & 2 & 12 & 15 & 88 & 0 & 0 & \\
\hline Rehabilitación & 1 & 6 & 16 & 94 & 0 & 0 & \\
\hline Ortodoncia & 0 & 0 & 21 & 81 & 5 & 19 & \\
\hline Odontopediatría & 3 & 18 & 14 & 82 & 0 & 0 & $\mathrm{p}=0,086$ \\
\hline \multicolumn{8}{|l|}{ Número de citas } \\
\hline 1 a 2 citas & 2 & 13 & 13 & 87 & 0 & 0 & \\
\hline 3 a 5 citas & 2 & 10 & 17 & 90 & 0 & 0 & \\
\hline 6 a mas citas & 2 & 4 & 44 & 85 & 6 & 12 & $\mathrm{p}=0,338$ \\
\hline \multicolumn{8}{|c|}{ Distrito de residencia } \\
\hline Cono norte & 2 & 3 & 57 & 92 & 3 & 5 & \\
\hline Cono sur & 1 & 6 & 15 & 83 & 2 & 11 & \\
\hline Centro & 3 & 30 & 6 & 60 & 1 & 10 & $\mathrm{p}=0,023$ \\
\hline
\end{tabular}


en Brasil el 2006 donde buena parte de los pacientes accede cada vez más a internet en busca de información que les permita conversar con sus médicos, lo cual demuestra un cambio de actitud y empoderamiento de su proceso de decisión sobre su salud, esto obliga al profesional a mantenerse siempre actualizado.

En relación a la autonomía del paciente y las covariables: sexo, edad, grado de instrucción, no se encontraron diferencias significativas, contrario a lo encontrado en el estudio de Benedetti (10) que observó, que pese, a que el número de varones encuestados era menor al de mujeres, nota la tendencia de que los varones sean más autónomos que las mujeres, en la capacidad de elegir opciones de tratamiento.

En relación a la autonomía y la persona que respondió al cuestionario, se observó que los pacientes tienen una mejor autonomía en la toma de decisiones que los tutores. Por otro lado se vio una relación con el lugar de residencia, debido a que la mayor parte de la población que acude a la clínica reside en el cono norte, dentro de la cual podrían estar involucrados algunos aspectos como nivel socioeconómico y dependencia económica del paciente como refieren en otro estudio (10).

En esta dimensión no se encontró influencia de las covariables edad y parentesco del operador.

\section{Dimensión Valores:}

Los valores del profesional se evaluaron en función de la puntualidad, responsabilidad, ambiente de confianza con el profesional; trato del personal auxiliar y la percepción de una atención y respeto con equidad.

Los resultados arrojaron en su mayoría una percepción de muy favorable y favorable, lo cual concuerda con los hallazgos de Simón (6) en España en los que los pacientes encuestados refieren sentirse respetados y escuchados por profesional dándole importancia capital al trato recibido.

Los sujetos encuestados provinieron de la misma fuente potencial de sujetos elegibles y la selección fue al azar evitando así el sesgo de selección. La estrategia de utilizar encuestas anónimas permite que el paciente brinde una información real puesto que le resta el miedo para expresarse libremente.

Los resultados pueden generali-

Tabla 5. Resultado de la Dimensión Valores del Profesional según covariables.

\begin{tabular}{|c|c|c|c|c|c|c|c|}
\hline & \multicolumn{2}{|c|}{ Muy Favorable } & \multicolumn{2}{|c|}{ Favorable } & \multicolumn{2}{|c|}{ Desfavorable } & \\
\hline & $\mathrm{n}$ & $\%$ & $\mathrm{n}$ & $\%$ & $\mathrm{n}$ & $\%$ & \\
\hline \multicolumn{8}{|l|}{ Sexo } \\
\hline Masculino & 9 & 33 & 17 & 63 & 1 & 4 & \\
\hline Femenino & 33 & 52 & 30 & 48 & 0 & 0 & $\mathrm{p}=0,098$ \\
\hline \multicolumn{8}{|l|}{ Edad } \\
\hline $18-24$ & 7 & 41 & 9 & 53 & 1 & 6 & \\
\hline $25-50$ & 23 & 44 & 29 & 56 & 0 & 0 & \\
\hline $51-74$ & 12 & 57 & 9 & 43 & 0 & 0 & $\mathrm{p}=0,245$ \\
\hline \multicolumn{8}{|c|}{ Grado de instrucción } \\
\hline Primaria & 0 & 0 & 3 & 100 & 0 & 0 & \\
\hline Secundaria & 10 & 48 & 11 & 52 & 0 & 0 & \\
\hline Técnica & 8 & 57 & 6 & 43 & 0 & 0 & \\
\hline Universidad & 24 & 47 & 27 & 51 & 1 & 2 & $\mathrm{p}=0,761$ \\
\hline \multicolumn{8}{|c|}{ Persona que informa } \\
\hline Paciente & 24 & 49 & 24 & 49 & 1 & 2 & \\
\hline Apoderado & 18 & 44 & 23 & 56 & 0 & 0 & $\mathrm{p}=0,555$ \\
\hline \multicolumn{8}{|c|}{ Servicio de atención } \\
\hline Cirugía & 2 & 22 & 7 & 78 & 0 & 0 & \\
\hline Periodoncia & 3 & 75 & 1 & 25 & 0 & 0 & \\
\hline Endodoncia & 11 & 65 & 6 & 35 & 0 & 0 & \\
\hline Rehabilitación & 10 & 63 & 7 & 38 & 0 & 0 & \\
\hline Ortodoncia & 8 & 31 & 17 & 65 & 1 & 4 & \\
\hline Odontopediatría & 8 & 47 & 9 & 53 & 0 & 0 & $\mathrm{p}=0,383$ \\
\hline \multicolumn{8}{|l|}{ Número de citas } \\
\hline 1 a 2 citas & 7 & 47 & 12 & 53 & 0 & 0 & \\
\hline 3 a 5 citas & 12 & 63 & 7 & 37 & 0 & 0 & \\
\hline 6 a mas citas & 23 & 44 & 28 & 54 & 1 & 2 & $\mathrm{p}=0,379$ \\
\hline
\end{tabular}


zarse a la población de pacientes que acuden a la unidad de posgrado de la Clínica Estomatológica Central de la UPCH en el tiempo en que se llevó a cabo la investigación.

Se obtuvieron resultados bastante positivos, debido a que se observa que el proceso de consentimiento informado en el servicio de posgrado es favorable, lo que quiere decir que los pacientes tienen una percepción bastante clara y precisa de los tratamientos que se les van a realizar.

\section{Referencias bibliográficas}

1. Palomer L. Consentimiento informado en odontología: un análisis teórico-práctico. Acta Bioeth. 2009; 15(1):100-5.

2. Miguel R, Zemel M. El consentimiento informado en odontólogos residentes de la Facultad de Odontología de la Universidad Nacional de La Plata. Acta Bioeth. 2006; 12(1):81-7.

3. Triana J. La ética: un problema para el odontólogo. Acta Bioeth. 2006; 12(1):75-80.

4. Matabuena C. Salud 02 y Metanoia: cambios en La rela- ción médico paciente. Bitácora Médica. (on line) 2010; 2(21). Disponible en: http:// bitacoramedica.com/weblog/ 2010/06/salud-2-0-y-metanoiacambios-en-la-relacion-medicopaciente/.acceso en: 30 mayo de 2009.

5. García F. El odontólogo frente al consentimiento informado: ¿Qué hacer? En: Cardozo C, Rodríguez E, Lolas F, Quezada A, et al. Ética y odontología una introducción. Centro Interdisciplinario de Estudios de Bioética. Chile; 2007: 240.

6. Simón P, Barrio IM, Sánchez CM, Tamayo MI, Molina A, Suess A, Jiménez A. Satisfacción de los pacientes con el proceso de información, consentimiento y toma de decisiones durante la hospitalización. Rev An Sis Sanit Navar. 2007; 30 (2): 35-42

7. Martín MC. Diseño y validación de cuestionarios. Matronas Profesión. 2004; (5):23-9.

8. Campos A, Oviedo H. Propiedades psicométricas de una escala. Rev Salud Pública. 2008;
10(5):831-39.

9. Guix J, Balaña F, Carbonell JM, Pérez R, Surroca RM, Nualartl L. Cumplimiento y percepción del consentimiento informado en un sector sanitario de Cataluña. Rev Esp Salúd Pública. 1999; 73(6): 20-8.

10.De Benedetti ME, Pastor SM, Bandrés MP. Evaluación de la autonomía del paciente en el proceso de la toma de decisiones médicas, en pacientes hospitalizados en el servicio de medicina interna del Hospital Nacional Arzobispo Loaiza. Rev Med Hered. 2006; (17): 21-27.

11. Mortensen MG, Kiyak HA, Omnell L. Patient and parent understanding of informed consent in orthodontics. Am.J.Orthod Dentofacial Orthop.2003; 124:541-50.

12. Silva WD. Avaliação de impactos do uso da Internet na relação médico-paciente. (tese MG). São Paulo: Universidade de São Paulo. Faculdade de Saúde Pública. 2006. 132p. 